\title{
TOPOLOGICAL PROPERTIES OF $q$-CONVEX SETS
}

\author{
GUIDO LUPACCIOLU
}

\begin{abstract}
We discuss the topological properties of a certain class of compact sets in a $q$-complete complex manifold $M$. These sets-which we call $q$ convex in $M$-include, for $q=0$, the $\mathscr{O}(M)$-convex compact sets in a Stein manifold. Then we show applications of the topological results to the subjects of removable singularities for $\bar{\partial}_{b}$.
\end{abstract}

\section{INTRODUCTION}

It is known that, if $K \subset \mathbb{C}^{n}$ is a polynomially convex compact set, the Čech cohomology spaces $H^{r}(K, \mathbb{C})=0$ for $r \geq n$ (see [12, Theorem 2.7.12]). This follows, by an inductive limit consideration, from the parallel result for Runge domains, due to Serre [16] (since $K$ has a neighborhood basis of Runge domains), and was also proved by Browder [6].

From the above property of polynomially convex sets Alexander [2] derived, by merely topological considerations, the following result about the polynomial hull $\hat{X}$ of a compact set $X$ in the boundary of the open unit ball $\mathbb{B}$ of $\mathbb{C}^{n}$, $n \geq 2$ : the Čech homology spaces $H_{i}(b \mathbb{B} \backslash X, \mathbb{C}) \cong H_{i}(\mathbb{B} \backslash \widehat{X}, \mathbb{C})$ for $i \leq n-2$, in particular $b \mathbb{B} \backslash X$ and $\mathbb{B} \backslash \widehat{X}$ have the same number (not necessarily finite) of connected components. ${ }^{1}$

Moreover recently Alexander and Stout [3] proved, by arguments of different nature, that the latter property extends to a more general context. The result of [3] can be stated as follows: Let $D$ be a relatively compact $C^{2}$-bounded strongly pseudoconvex domain in a Stein manifold $M(\operatorname{dim} M \geq 2)$, with $\bar{D}$ being $\mathscr{O}(M)$-convex, and $X$ a compact set in $b D$; then, if $\widehat{X}_{M}$ denotes the $\mathscr{O}(M)$-hull of $X, b D \backslash X$ and $D \backslash \widehat{X}_{M}$ have the same number of connected components.

In this paper we pursue the investigation about topological properties of this sort for a suitable class of compact sets in a $q$-complete complex manifold $M$, namely the compact sets which are $q$-convex in $M$, according to the definition given below. These sets are a quite natural generalization to the setting of a $q$-complete manifold of the $\mathscr{O}(M)$-convex sets in a Stein manifold.

Our results generalize and improve the above mentioned topological results and include a characterization of Behnke-Stein type of the $(n-1)$-convex compact sets in an arbitrary noncompact complex manifold.

Received by the editors November 13, 1990 and, in revised form, February 20, 1991.

1980 Mathematics Subject Classification (1985 Revision). Primary 32F10; Secondary 32E20.

${ }^{1}$ Some consequences of this fact are shown in [5]. 
Moreover we show applications relating to the subject of removable singularities for the tangential Cauchy-Riemann operator.

\section{1. q-CONVEX SETS}

Let $M$ be a noncompact complex manifold of dimension $n \geq 2$ (connected, with a countable topology).

As is well known, $M$ is said to be $q$-complete $(0 \leq q \leq n-1)$ in case there exists a $C^{\infty}$ strongly $q$-plurisubharmonic proper function $\rho: M \rightarrow \mathbb{R}$. In particular $M$ is 0 -complete if and only if it is Stein, and every noncompact complex manifold is $(n-1)$-complete (see [9]). We also recall that $q$-completeness entails the vanishing of the Dolbeault cohomology spaces $H_{\bar{\partial}}^{r}, s(M), r \geq 0$, $s>q$ and of the de Rham cohomology spaces $H_{D R}^{j}(M, \mathbb{C}), j>n+q$ (see [4, 20]).

If $M$ is $q$-complete and $K \subset M$ is a compact set, let us say that $K$ is $q$ convex in $M$ if the following holds: Given arbitrarily an open neighborhood $\omega$ of $K$, it is possible to find a $C^{\infty}$ strongly $q$-plurisubharmonic proper function $u: M \rightarrow \mathbb{R}$ such that $K \subset\{x \in M: u(x)<0\} \subset \subset \omega$.

We have already considered this notion of $q$-convexity previously [13]. It appears as a generalization to a $q$-complete manifold of the standard notion of $\mathscr{O}(M)$-convexity relative to a compact set in a Stein manifold. In fact, if $M$ is Stein, a compact set $K \subset M$ is 0 -convex according to the above definition if and only if $K=\widehat{K}_{M}(=\mathscr{O}(M)$-hull of $K$ ) (see [12, Theorems 5.1.6 and 5.2.10]).

We point out that, though the $(n-1)$-completeness condition does not impose any restriction to a noncompact complex manifold $M$, not every compact set $K \subset M$ is $(n-1)$-convex in $M$ (see Theorem 2 below).

Our first result is the following generalization of the result of Serre and Browder mentioned at the beginning.

Theorem 1. Let $M$ be a q-complete manifold of dimension $n \geq 2(0 \leq q \leq$ $n-1)$ and $K \subset M$ a compact set which is q-convex in $M$. Then the restriction map

$$
H^{n+q}(M, \mathbb{C}) \rightarrow H^{n+q}(K, \mathbb{C})
$$

is surjective, moreover $H^{r}(K, \mathbb{C})=0$ for $r>n+q$.

Proof. The basic result on which Theorem 1 depends is the Andreotti-Grauert approximation theorem [4, Theorem 12] (see also [11, Theorem 12.11]). This implies the following fact:

(*) Let $u: M \rightarrow \mathbb{R}$ be a $C^{\infty}$ strongly $q$-plurisubharmonic proper function and set $M_{0}(u)=\{x \in M: u(x)<0\}$; then, if $W$ is any holomorphic vector bundle on $M$ of finite rank, the restriction maps

$$
Z_{\bar{\partial}}^{r, s}(M, W) \rightarrow Z_{\bar{\partial}}^{r, s}\left(M_{0}(u), W\right), \quad r \geq 0, s \geq q,
$$

have dense images, with respect to the natural Fréchet-Schwartz topologies of the spaces of $C^{\infty} W$-valued differential forms.

Moreover $M_{0}(u)$ is $q$-complete, since the function $(-u)^{-1}: M_{0}(u) \rightarrow \mathbb{R}$ is strongly $q$-plurisubharmonic and proper.

Then, in view of $(*)$ (for $W=M \times \mathbb{C}$ ) and of the vanishing of $H_{\bar{\partial}}^{r, s}(M)$ and $H_{\bar{\partial}}^{r}, s\left(M_{0}(u)\right)$ for $r \geq 0$ and $s>q$, a standard argument (see $[19, \S 6]$ ) 
shows that the following is true too:

$(* *)$ The restriction map $H_{D R}^{n+q}(M, \mathbb{C}) \rightarrow H_{D R}^{n+q}\left(M_{0}(u), \mathbb{C}\right)$ has dense image and $H_{D R}^{r}\left(M_{0}(u), \mathbb{C}\right)=0$ for $r>n+q$.

Now, as $K$ is $q$-convex in $M$, we can find a neighborhood basis of $K$ of open sets of the form $M_{0}(u)$, such that, in addition, 0 is a regular value of $u$. For every such $M_{0}(u), \overline{M_{0}(u)}$ is a (possibly disconnected) compact $C^{\infty}$ manifold with boundary, and hence the Čech (or singular) cohomology spaces $H^{j}\left(\overline{M_{0}(u)}, \mathbb{C}\right)$ and $H^{j}\left(b M_{0}(u), \mathbb{C}\right), j \geq 0$, are finite dimensional (see [7, p. 103]). Then, by the cohomology sequence with compact supports

$$
\cdots \rightarrow H_{c}^{j}\left(M_{0}(u), \mathbb{C}\right) \rightarrow H^{j}\left(\overline{M_{0}(u)}, \mathbb{C}\right) \rightarrow H^{j}\left(b M_{0}(u), \mathbb{C}\right) \rightarrow \cdots,
$$

also the spaces $H_{c}^{j}\left(M_{0}(u), \mathbb{C}\right), j \geq 0$, are finite dimensional, and by the Poincaré duality and the de Rham isomorphism, so are the spaces $H_{D R}^{2 n-j}\left(M_{0}(u), \mathbb{C}\right), j \geq$ 0 , too. Therefore, since every vector subspace of a finite-dimensional Hausdorff vector space is closed (see [10, p. 38]), (**) implies that the restriction map $H_{D R}^{n+q}(M, \mathbb{C}) \rightarrow H_{D R}^{n+q}\left(M_{0}(u), \mathbb{C}\right)$ is in fact surjective (at least in the case under consideration that 0 is a regular value of $u$ ).

Then the conclusion follows at once by the de Rham isomorphism and an inductive limit consideration. Q.E.D.

Our next theorem gives a characterization of $(n-1)$-convex compact sets in an arbitrary noncompact complex manifold, and, in particular, shows that for $q=n-1$ the converse of Theorem 1 is true too.

Theorem 2. Let $M$ be a noncompact complex manifold of dimension $n \geq 2$ and $K \subset M$ a compact set. The following conditions on $K$ are equivalent:

(a) $K$ is $(n-1)$-convex in $M$;

(b) The restriction map $H^{2 n-1}(M, \mathbb{C}) \rightarrow H^{2 n-1}(K, \mathbb{C})$ is surjective;

(c) $M \backslash K$ has no relatively compact connected components;

(d) Every $C^{\infty}$ form in $Z_{\bar{\partial}}^{r}{ }^{n-1}(K, W)$ can be uniformly approximated on $K$ together with all derivatives of the coefficients by $C^{\infty}$ forms in $Z_{\bar{\partial}}^{r, n-1}(M, W)$ $(r \geq 0, W$ any holomorphic vector bundle on $M$ of finite rank).

Moreover, in case $H^{2 n-1}(M, \mathbb{C})=0$, the above conditions are also equivalent to the following one:

(e) $M \backslash K$ is connected.

Proof. We first prove that $(\mathrm{a}) \Rightarrow(\mathrm{b}) \Rightarrow(\mathrm{c})$ and that $(\mathrm{d}) \Rightarrow(\mathrm{b})$. That $(\mathrm{a}) \Rightarrow(\mathrm{b})$ follows at once from Theorem 1 . Then assume that $(\mathrm{b})$ is valid. Since $M$ is noncompact, $H^{2 n}(M, \mathbb{C})=0$ (see [7, p. 260]), hence the relative cohomology sequence

$$
\cdots \rightarrow H^{j}(M, \mathbb{C}) \rightarrow H^{j}(K, \mathbb{C}) \rightarrow H^{j+1}(M, K ; \mathbb{C}) \rightarrow \cdots
$$

implies that $H^{2 n}(M, K ; \mathbb{C})=0$. On the other hand

$$
H^{2 n}(M, K ; \mathbb{C}) \cong H_{\Phi}^{2 n}(M \backslash K, \mathbb{C}),
$$

where $\Phi$ is the paracompactifying family of supports in $M \backslash K$ made up by the subsets of $M \backslash K$ which are closed in $M$ (see [8, p. 235]). Now, if there existed a relatively compact connected component $U$ of $M \backslash K, H_{\Phi \mid U}^{2 n}(U, \mathbb{C})=$ $H_{c}^{2 n}(U, \mathbb{C}) \cong \mathbb{C}$ would be a direct summand of $H_{\Phi}^{2 n}(M \backslash K, \mathbb{C})$, and the latter would not be zero. Therefore (b) $\Rightarrow$ (c) . 
The proof that $(d) \Rightarrow(b)$ is essentially the same as the proof of Theorem 1 , using condition (d) in place of the Andreotti-Grauert approximation theorem. The vanishing of $H_{\bar{\partial}}^{r, s}(M)$ and $H_{\bar{\partial}}^{r, s}(K)$ for $r \geq 0$ and $s \geq n$, which is also needed, follows from the quoted result of [9] (see also below).

On the other hand, by resorting again to the Andreotti-Grauert approximation theorem, we get immediately, via an inductive limit consideration, that (a) $\Rightarrow$ (d).

Next let us prove that $(c) \Rightarrow(a)$, which will conclude the proof that $(a)$, (b), (c), and (d) are equivalent. Thus assume that (c) is valid. Choose a $C^{\infty}$ Hermitian metric $G$ on $M$, and, in accordance with Greene-Wu [9], define a Laplacian $\Delta_{0}$ on $M$ by setting $\Delta_{0}=\sum_{\alpha, \beta}^{1, n} G^{\alpha \beta} \partial^{2} / \partial z_{\alpha} \partial \bar{z}_{\beta}$ for every allowable system $\left(z_{1}, \ldots, z_{n}\right)$ of local complex coordinates. It is proved in [9], using the Lax-Malgrange approximation theorem of Runge type applied to $\Delta_{0}$, that there exists a nonnegative $C^{\infty}$ strongly $\Delta_{0}$-subharmonic proper function $\tau_{0}: M \rightarrow$ $\mathbb{R}$; and since $\Delta_{0} \tau_{0}$ is the trace of the Levi form of $\tau_{0}$, the latter is strongly $(n-1)$-plurisubharmonic (which shows $M$ to be $(n-1)$-complete). Moreover, after adding a constant, we may assume that $\tau_{0}$ is strictly positive.

We are to prove that, given an open neighborhood $\omega$ of $K$, it is possible to find a $C^{\infty}$ strongly $(n-1)$-plurisubharmonic proper function $u: M \rightarrow \mathbb{R}$ such that $K \subset\{u<0\} \subset \subset \omega$.

Let $\omega_{1}$ be an open neighborhood of $K$ with $\omega_{1} \subset \subset \omega$, and choose a positive real number $R$ large enough so that $\omega_{1} \subset M_{R}\left(\tau_{0}\right)=\left\{x \in M: \tau_{0}(x)<R\right\}$.

Since $M \backslash K$ has no relatively compact connected components, if $x$ is any point in $M \backslash K$ we can, by the Lax-Malgrange theorem for $\Delta_{0}$ again, find a $\Delta_{0}$-harmonic function $h: M \rightarrow \mathbb{R}$ with $h(x)>1$ and $h<1$ on $K$. Therefore, as $\overline{M_{R}\left(\tau_{0}\right)} \backslash \omega_{1}$ is compact, we can choose finitely many $\Delta_{0}$-harmonic functions $h_{1}, \ldots, h_{N}: M \rightarrow \mathbb{R}$ which are $<1$ on $K$, so that $\max \left\{h_{1}(x), \ldots, h_{N}(x)\right\}>1$ for every $x \in \overline{M_{R}\left(\tau_{0}\right)} \backslash \omega_{1}$. Set moreover $h_{0}=\tau_{0} / R$. Clearly, we can choose a positive integer $r$ large enough so that the function $u=h_{0}^{2 r}+\cdots+h_{N}^{2 r}-1$ is $<0$ on $K$ and is $>0$ on $M \backslash \omega_{1}$. Hence $u: M \rightarrow \mathbb{R}$ is a $C^{\infty}$ proper function such that $K \subset\{u<0\} \subset \subset \omega$. There remains only to show that $u$ is strongly $(n-1)$-plurisubharmonic. As a matter of fact we have, in the domain of every system of local coordinates $\left(z_{1}, \ldots, z_{n}\right)$,

$$
\Delta_{0} u=2 r \sum_{j=0}^{N}\left\{(2 r-1) h_{j}^{2(r-1)} \sum_{\alpha, \beta}^{1, n} G^{\alpha \beta} \frac{\partial h_{j}}{\partial z_{\alpha}} \frac{\overline{\partial h_{j}}}{\partial z_{\beta}}+h_{j}^{2 r-1} \Delta_{0} h_{j}\right\},
$$

and since $h_{0}, \Delta_{0} h_{0}$ are strictly positive, $\Delta_{0} h_{j}=0, j=1, \ldots, N$, and $\left(G^{\alpha \beta}(z)\right)$ is a positive definite Hermitian matrix, it follows that $\Delta_{0} u$ is strictly positive. Thus $u$ is strongly $\Delta_{0}$-subharmonic, and consequently strongly $(n-1)$ plurisubharmonic, which concludes the proof that $(\mathrm{c}) \Rightarrow(\mathrm{a})$.

There remains to prove the last statement of the theorem. Since clearly (e) $\Rightarrow$ (c) , it suffices to show that, under the additional assumption $H^{2 n-1}(M, \mathbb{C})=0$, (b) $\Rightarrow(\mathrm{e})$. As a matter of fact we have $H^{2 n-1}(K, \mathbb{C})=0$, and therefore the exact cohomology sequence with compact supports

$$
\cdots \rightarrow H^{j}(K, \mathbb{C}) \rightarrow H_{c}^{j+1}(M \backslash K, \mathbb{C}) \rightarrow H^{j+1}(M, \mathbb{C}) \rightarrow \cdots
$$

implies that $H_{c}^{2 n}(M \backslash K, \mathbb{C}) \cong H_{c}^{2 n}(M, \mathbb{C}) \cong \mathbb{C}$, and this shows $M \backslash K$ to be connected. Q.E.D. 
The above theorem improves a result obtained previously by Silva [17], using different techniques, in the direction of extending to $n \geq 2$ the classical BehnkeStein theorem that characterizes the holomorphically convex compact sets in a noncompact Riemann surface. The result of [17] (generalized to complex spaces in [18]) is essentially equivalent to proving that (c) $\Leftrightarrow$ (d). An earlier approximation result of this type in the case $M=\mathbb{C}^{n}$ and $K=\bar{D}\left(D \subset \subset \mathbb{C}^{n}\right.$ a domain with smooth boundary) is due to Weinstock [21]. A proof that (c) $\Leftrightarrow$ (d) in the case $M=\mathbb{C}^{n}$ can also be found in [1].

\section{SePARATION PROPERTIES OF $q$-CONVEX SETS}

This section is devoted to discuss the topological properties of $q$-convex sets of the kind of the results of Alexander and of Alexander-Stout mentioned in the Introduction.

In the first place we have

Proposition 1. Let $M$ be a $(n-2)$-complete manifold of dimension $n \geq 2$, such that $H^{2 n-2}(M, \mathbb{C})=0, K \subset M$ a compact set which is $(n-2)$-convex in $M$ and $D \subset \subset M$ an open domain such that $b D \backslash K$ is a $(2 n-1)$-dimensional topological manifold. Then, provided $M \backslash(\bar{D} \cup K)$ is connected, ${ }^{2} H_{0}(b D \backslash K, \mathbb{C}) \cong$ $H_{0}(D \backslash K, \mathbb{C})$.

Proof. By Theorem $1, H^{j}(K, \mathbb{C})=0$ for $j \geq 2 n-2$, and, by Theorem 2 , $H^{2 n-1}(\bar{D} \cup K, \mathbb{C})=0$. Moreover $0=H^{2 n}(\bar{D}, \mathbb{C})=H^{2 n}(\bar{D} \cup K, \mathbb{C})$ because $M$ is noncompact. Therefore the Mayer-Vietoris cohomology sequence

$$
\cdots \rightarrow H^{j}(\bar{D} \cup K, \mathbb{C}) \rightarrow H^{j}(\bar{D}, \mathbb{C}) \oplus H^{j}(K, \mathbb{C}) \rightarrow H^{j}(\bar{D} \cap K, \mathbb{C}) \rightarrow \cdots
$$

implies that the restriction map $H^{j}(\bar{D}, \mathbb{C}) \rightarrow H^{j}(\bar{D} \cap K, \mathbb{C})$ is surjective for $j \geq 2 n-2$ and is an isomorphism for $j \geq 2 n-1$. Then the exact cohomology sequence with compact supports

$$
\cdots \rightarrow H_{c}^{j}(\bar{D} \backslash K, \mathbb{C}) \rightarrow H^{j}(\bar{D}, \mathbb{C}) \rightarrow H^{j}(\bar{D} \cap K, \mathbb{C}) \rightarrow \cdots
$$

gives $H_{c}^{j}(\bar{D} \backslash K, \mathbb{C})=0$ for $j \geq 2 n-1$. On the other hand there is also an exact cohomology sequence with compact supports

$$
\cdots \rightarrow H_{c}^{j}(D \backslash K, \mathbb{C}) \rightarrow H_{c}^{j}(\bar{D} \backslash K, \mathbb{C}) \rightarrow H_{c}^{j}(b D \backslash K, \mathbb{C}) \rightarrow \cdots,
$$

and hence it follows that $H_{c}^{2 n-1}(b D \backslash K, \mathbb{C}) \cong H_{c}^{2 n}(D \backslash K, \mathbb{C})$. Then, by the Poincaré duality, we also have $H_{0}(b D \backslash K, \mathbb{C}) \cong H_{0}(D \backslash K, \mathbb{C})$, which concludes the proof. Q.E.D.

Proposition 1 implies in particular

Corollary 1. Let $M$ be a Stein manifold of dimension $n \geq 2$, such that $H^{2 n-2}(M, \mathbb{C})=0,^{3} X \subset M$ a compact set and $D \subset \subset M$ an open domain. Let $\widehat{X}_{M}$ denote the $\mathscr{O}(M)$-hull of $X$, and assume that $b D \backslash \widehat{X}_{M}$ is a $(2 n-1)$ dimensional topological manifold. Then, provided $M \backslash\left(\bar{D} \cup \widehat{X}_{M}\right)$ is connected, $H_{0}\left(b D \backslash \widehat{X}_{M}, \mathbb{C}\right) \cong H_{0}\left(D \backslash \widehat{X}_{M}, \mathbb{C}\right)$.

\footnotetext{
${ }^{2}$ Note that, since $H^{2 n-1}(M, \mathbb{C})=0$ (due to $(n-2)$-completeness), in view of Theorem 2 applied to $\bar{D} \cup K$, this assumption is not more restrictive than the assumption that $M \backslash(\bar{D} \cup K)$ has no relatively compact connected components.

${ }^{3}$ Of course this condition does not impose any restriction to the Stein manifold $M$ if $n \geq 3$.
} 
Proof. Set $K=\widehat{X}_{M}$. Then $M$ is 0-complete, hence $(n-2)$-complete, and $K$ is 0 -convex in $M$, hence $(n-2)$-convex in $M$. Therefore the thesis follows at once from Proposition 1. Q.E.D.

We wish to point out that what Proposition 1 means is that the boundary of every connected component of $D \backslash K$ contains exactly one connected component of $b D \backslash K$; however saying that $b D \backslash K$ and $D \backslash K$ have the same number of connected components could be inappropriate, since this number need not be finite. To show an example, consider the open unit ball $\mathbb{B}$ in $\mathbb{C}^{n}, n \geq 2$, take a point $\zeta \in b \mathbb{B}$ and set

$$
X=\{\zeta\} \cup\left[b \mathbb{B} \cap\left(\bigcup_{k=1}^{\infty} b \mathbb{B}\left(\zeta, \frac{1}{k}\right)\right)\right],
$$

where $\mathbb{B}(\zeta, 1 / k)$ is the open ball with center $\zeta$ and radius $1 / k$. Then $X$ is a compact subset of $b \mathbb{B}$ such that $b \mathbb{B} \backslash X=b \mathbb{B} \backslash \widehat{X}$ is made up by infinitely many connected components.

For a further investigation on the separation properties of $q$-convex sets we need the following proposition.

Proposition 2. Let $M$ be a q-complete manifold of dimension $n \geq 2(0 \leq$ $q \leq n-1), K \subset M$ a compact set which is $q$-convex in $M$ and $F \subset M a$ closed set endowed with a neighborhood basis of p-complete open sets $(0 \leq p \leq$ $n-1-q)$. Then the restriction map $H^{n+p+q}(F, \mathbb{C}) \rightarrow H^{n+p+q}(F \cap K, \mathbb{C})$ is surjective, moreover $H^{r}(F \cap K, \mathbb{C})=0$ for $r>n+p+q$.

Proof. Let $\Omega_{1}$ be a $p$-complete open neighborhood of $F$ and $\omega \subset \subset M$ an open neighborhood of $K$. We shall prove that there exists a $C^{\infty}$-bounded $(p+q)$-complete open neighborhood $\Omega_{2} \subset \Omega_{1} \cap \omega$ of $F \cap K$ such that the restriction map $H^{n+p+q}\left(\Omega_{1}, \mathbb{C}\right) \rightarrow H^{n+p+q}\left(\Omega_{2}, \mathbb{C}\right)$ is onto. By the $(p+q)$ completeness of $\Omega_{2}$, we shall also have $H^{r}\left(\Omega_{2}, \mathbb{C}\right)=0$ for $r>n+p+q$, and the conclusion will then follow by an inductive limit consideration, in view of the fact that, as $\Omega_{1}$ and $\omega$ range through neighborhood bases of $F$ and $K$ respectively, $\Omega_{1} \cap \omega$ ranges through a neighborhood basis of $F \cap K$, and hence so does $\Omega_{2}$ too.

Since $\Omega_{1}$ is $p$-complete, there exists a $C^{\infty}$ strongly $p$-plurisubharmonic proper function $\varphi: \Omega_{1} \rightarrow \mathbb{R}$ and, after adding a constant, we may assume that $F \cap K \subset\{\varphi<0\}$. Moreover, since $K$ is $q$-convex, there exists a $C^{\infty}$ strongly $q$-plurisubharmonic proper function $u: M \rightarrow \mathbb{R}$ such that $K \subset\{u<0\} \subset \subset \omega$. Consider the functions $\exp (\varphi): \Omega_{1} \rightarrow \mathbb{R}$ and $\exp (u): M \rightarrow \mathbb{R}$. Since $\exp (\varphi)<$ 1 on $F \cap K, \exp (u)<1$ on $K$ and $\exp (\varphi) \geq 1$ on $\Omega_{1} \backslash\{\varphi<0\}, \exp (u)>1$ on $M \backslash \omega$, we can choose a positive integer $N$ large enough so that

$$
\exp (N \varphi)+\exp (N u) \begin{cases}<1, & \text { on } F \cap K, \\ >1, & \text { on } \Omega_{1} \backslash(\{\varphi<0\} \cap \omega) .\end{cases}
$$

Then choose a regular value $\alpha \leq 1$ of $\exp (N \varphi)+\exp (N u)$ such that $\exp (N \varphi)+$ $\exp (N u)<\alpha$ on $F \cap K$ and set

$$
\rho=\exp (N \varphi)+\exp (N u)-\alpha, \quad \Omega_{2}=\left\{x \in \Omega_{1}: \rho(x)<0\right\} .
$$

Clearly $F \cap K \subset \Omega_{2} \subset \Omega_{1} \cap \omega$ and $\Omega_{2}$ is $C^{\infty}$-bounded.

Now, as one can easily check, the function $\rho: \Omega_{1} \rightarrow \mathbb{R}$ is strongly $(p+q)$ plurisubharmonic and proper, and the same is true of the function $(-\rho)^{-1}: \Omega_{2}$ 
$\rightarrow \mathbb{R}$. Therefore $\Omega_{1}$ and $\Omega_{2}$ are both $(p+q)$-complete and $\Omega_{2}=\left[\Omega_{1}\right]_{0}(\rho)=$ $\left\{x \in \Omega_{1}: \rho(x)<0\right\}$. It follows, by the same reasoning as in the proof of Theorem 1, that the restriction map $H^{n+p+q}\left(\Omega_{1}, \mathbb{C}\right) \rightarrow H^{n+p+q}\left(\Omega_{2}, \mathbb{C}\right)$ is surjective, which gives the desired conclusion. Q.E.D.

Now we can prove the following refinement of Proposition 1.

Proposition 3. Let $M$ be a q-complete manifold of dimension $n \geq 2(0 \leq$ $q \leq n-2), K \subset M$ a compact set which is q-convex in $M$ and $D \subset \subset M$ an open domain such that $\bar{D}$ has a neighborhood basis of p-complete open sets $(0 \leq p \leq n-2-q)$ and $b D \backslash K$ is a topological $(2 n-1)$-dimensional manifold. Then $H_{i}(M \backslash(\bar{D} \cup K), \mathbb{C}) \cong H_{i}(M, \mathbb{C})$ and $H_{i}(b D \backslash K, \mathbb{C}) \cong H_{i}(D \backslash K, \mathbb{C})$ for $i \leq n-2-(p+q)$.

Proof. Consider the Mayer-Vietoris cohomology sequence

$$
\cdots \rightarrow H^{j}(\bar{D} \cup K, \mathbb{C}) \rightarrow H^{j}(\bar{D}, \mathbb{C}) \oplus H^{j}(K, \mathbb{C}) \rightarrow H^{j}(\bar{D} \cap K, \mathbb{C}) \rightarrow \cdots
$$

By Proposition 2 the homomorphism

$$
H^{n+p+q}(\bar{D}, \mathbb{C}) \oplus H^{n+p+q}(K, \mathbb{C}) \rightarrow H^{n+p+q}(\bar{D} \cap K, \mathbb{C})
$$

is surjective, and $H^{j}(\bar{D} \cap K, \mathbb{C})=0$ for $j>n+p+q$. Since moreover $0=H^{j}(\bar{D}, \mathbb{C})=H^{j}(K, \mathbb{C})$ for $j>n+p+q$, it follows that $H^{j}(\bar{D} \cup K, \mathbb{C})=0$ for $j>n+p+q$. Then consider the cohomology sequence with compact supports

$$
\cdots \rightarrow H_{c}^{j}(M \backslash(\bar{D} \cup K), \mathbb{C}) \rightarrow H_{c}^{j}(M, \mathbb{C}) \rightarrow H^{j}(\bar{D} \cup K, \mathbb{C}) \rightarrow \cdots .
$$

This implies, in view of the above,

$$
H_{c}^{j}(M \backslash(\bar{D} \cup K), \mathbb{C}) \cong H_{c}^{j}(M, \mathbb{C}) \quad \text { for } j \geq n+p+q+2 .
$$

Next consider the cohomology sequence with compact supports

$$
\cdots \rightarrow H_{c}^{j}(\bar{D} \backslash K, \mathbb{C}) \rightarrow H^{j}(\bar{D}, \mathbb{C}) \rightarrow H^{j}(\bar{D} \cap K, \mathbb{C}) \rightarrow \cdots .
$$

Since, by Proposition 2, the homomorphism $H^{n+p+q}(\bar{D}, \mathbb{C}) \rightarrow H^{n+p+q}(\bar{D} \cap$ $K, \mathbb{C})$ is surjective and $H^{j}(\bar{D} \cap K, \mathbb{C})=0$ for $j>n+p+q$, it follows that $H_{c}^{j}(\bar{D} \backslash K, \mathbb{C})=0$ for $j \geq n+p+q+1$. Then the cohomology sequence with compact supports

$$
\cdots \rightarrow H_{c}^{j}(D \backslash K, \mathbb{C}) \rightarrow H_{c}^{j}(\bar{D} \backslash K, \mathbb{C}) \rightarrow H_{c}^{j}(b D \backslash K, \mathbb{C}) \rightarrow \cdots
$$

implies

$$
H_{c}^{j}(b D \backslash K, \mathbb{C}) \cong H_{c}^{j+1}(D \backslash K, \mathbb{C}) \text { for } j \geq n+p+q+1 .
$$

Finally from $(*)$ and $(* *)$ we get, via the Poincare duality,

$$
H_{2 n-j}(M \backslash(\bar{D} \cup K), \mathbb{C}) \cong H_{2 n-j}(M, \mathbb{C})
$$

for $j \geq n+p+q+2$, and $H_{2 n-1-j}(b D \backslash K, \mathbb{C}) \cong H_{2 n-j-1}(D \backslash K, \mathbb{C})$ for $j \geq$ $n+p+q+1$. This is the desired conclusion. Q.E.D.

Proposition 3 implies in particular the following refinement of Corollary 1: 
Corollary 2. Let $M$ be a Stein manifold of dimension $n \geq 2, X \subset M a$ compact set and $D \subset \subset M$ an open domain such that $\bar{D}$ has a neighborhood basis of $p$-complete open sets $(0 \leq p \leq n-2)$ and $b D \backslash \widehat{X}_{M}$ is a $(2 n-1)$ dimensional topological manifold. Then $H_{i}\left(M \backslash\left(\bar{D} \cup \widehat{X}_{M}\right), \mathbb{C}\right) \cong H_{i}(M, \mathbb{C})$ and $H_{i}\left(b D \backslash \widehat{X}_{M}, \mathbb{C}\right) \cong H_{i}\left(D \backslash \widehat{X}_{M}, \mathbb{C}\right)$ for $i \leq n-2-p$.

Proof. It is a straightforward consequence of Proposition 3 for $q=0$ and $K=\widehat{X}_{M}$. Q.E.D.

Corollary 2 improves the results of [2 and 3] mentioned in the Introduction.

\section{REMOVABLE SINGULARITIES OF $\bar{\partial}_{b}$}

The above Propositions 1 and 3 and Corollaries 1 and 2 are of interest in the subject of removable singularities of the tangential Cauchy-Riemann operator $\bar{\partial}_{b}$. We proved the following theorem in this area [13, Theorem 2.3].

Let $M$ be a $(n-2)$-complete manifold of dimension $n \geq 2, D \subset \subset M$ an open domain and $E \subset M$ a compact set which is $(n-2)$-convex in $M$. Assume that $b D \backslash E$ is connected and of class $C^{1}$. Then every continuous $C R$-function $f$ on $b D \backslash E$ extends continuously to a function $F \in C^{0}(\bar{D} \backslash E) \cap \mathscr{O}(D \backslash E)$.

Now, by a direct inspection of the proof of this theorem, it appears that the connectedness of $b D \backslash E$ is not necessary; what is really needed in the proof is that $M \backslash(\bar{D} \cup E)$ is connected and every connected component of $b D \backslash E$ is the whole boundary, in $M \backslash E$, of a connected component of $D \backslash E$ (the latter being also necessary). Therefore, in view of Propositions 1 and 3, the following two other versions of the above-mentioned theorem are valid too.

Theorem 3. Let $M$ be $a(n-2)$-complete manifold of dimension $n \geq 2$ such that $H^{2 n-2}(M, \mathbb{C})=0, D \subset \subset M$ an open domain and $E \subset M$ a compact set which is $(n-2)$-convex in $M$. Assume that $b D \backslash E$ is of class $C^{1}$ and that $M \backslash(\bar{D} \cup E)$ is connected. Then every continuous $C R$-function $f$ on $b D \backslash E$ extends continuously to a function $F \in C^{0}(\bar{D} \backslash E) \cap \mathscr{O}(D \backslash E)$.

Theorem 4. Let $M$ be a q-complete manifold of dimension $n \geq 2(0 \leq q \leq$ $n-2), D \subset \subset M$ an open domain and $E \subset M$ a compact set which is $q$-convex in $M$. Assume that $b D \backslash E$ is of class $C^{1}$ and that $\bar{D}$ has a neighborhood basis of $(n-2-q)$-complete open sets. Then every continuous $C R$-function $f$ on $b D \backslash E$ extends continuously to a function $F \in C^{0}(\bar{D} \backslash E) \cap \mathscr{O}(D \backslash E)$.

Let us point out that the result of [13] recalled above is actually more general than is stated here, as it concerns not only $C R$-functions, but other kinds of $C R$-objects on $b D \backslash E$ as well, under suitable smoothness assumptions on $b D \backslash E$. The parallel more general versions of Theorems 3 and 4 are valid too.

It is worth noticing the following particular case of both Theorems 3 and 4 (in view of Corollaries 1 and 2 too).

Corollary 3. Let $D \subset \subset \mathbb{C}^{n}, n \geq 2$, be an open domain and $X \subset \mathbb{C}^{n}$ a compact set. Assume that $b D \backslash \widehat{X}^{4}$ is of class $C^{1}$ and $\mathbb{C}^{n} \backslash(\bar{D} \cup \widehat{X})$ is connected. Then every continuous $C R$-function $f$ on $b D \backslash \widehat{X}$ extends continuously to a function

\footnotetext{
${ }^{4} \widehat{X}=$ polynomial hull of $X$.
} 
$F \in C^{0}(\bar{D} \backslash \widehat{X}) \cap \mathscr{O}(D \backslash \widehat{X})$. Moreover the connectedness of $\mathbb{C}^{n} \backslash(\bar{D} \cup \widehat{X})$ holds in particular if $\bar{D}$ has a neighborhood basis of $(n-2)$-complete open sets.

This corollary shows that the $\bar{\partial}_{b}$-removability of polynomially convex sets is valid under less restrictive assumptions than it was proved in [14 and 15].

\section{REFERENCES}

1. L. A. Aizenberg and Sh. Dautov, Differential forms orthogonal to holomorphic functions or forms, and their properties, "Nauka", Novosibirsk, 1975; English transl., Amer. Math. Soc., Providence, R.I., 1983.

2. H. Alexander, A note on polynomial hulls, Proc. Amer. Math. Soc. 33 (1972), 389-391.

3. H. Alexander and E. L. Stout, A note on hulls, Bull. London Math. Soc. (3) 22 (1990), 258-260.

4. A. Andreotti and $\mathrm{H}$. Grauert, Théorèmes de finitude pour la cohomologie des espaces complexes, Bull. Soc. Math. France 90 (1962), 193-259.

5. R. F. Basener, Complementary components of polynomial hulls, Proc. Amer. Math. Soc. 69 (1978), 230-232.

6. A. Browder, Cohomology of maximal ideal spaces, Bull. Amer. Math. Soc. 67 (1961), 515516.

7. A. Dold, Lectures on algebraic topology, Springer-Verlag, Berlin, Heidelberg and New York, 1972.

8. R. Godement, Topologie algébrique et théorie des faisceaux, Hermann, Paris, 1973.

9. R. E. Greene and $\mathrm{H}$. Wu, Whitney's imbedding theorem by solutions of elliptic equations and geometric consequences, Proc. Sympos. Pure Math., vol. 27, Part 2, Amer. Math. Soc., Providence, R.I., 1975, pp. 287-296.

10. A. Grothendieck, Topological vector spaces, Gordon and Breach, New York, London and Paris, 1973.

11. G. M. Henkin and J. Leiterer, Andreotti-Grauert theory by integral formulas, AkademieVerlag, Berlin, 1988.

12. L. Hörmander, An introduction to complex analysis in several variables, North-Holland, Amsterdam, 1973.

13. G. Lupacciolu, Some global results on extension of CR-objects in complex manifolds, Trans. Amer. Math. Soc. 321 (1990), 761-774.

14. _ A theorem on holomorphic extension of CR-functions, Pacific J. Math. 124 (1986), 177-191.

15. J. P. Rosay and E. L. Stout, Radò's theorem for CR-functions, Proc. Amer. Math. Soc. 106 (1989), 1017-1026.

16. J. P. Serre, Une propriété topologique des domaines de Runge, Proc. Amer. Math. Soc. 6 (1955), 133-134.

17. A. Silva, $A$ n-Runge approximation theorem, Boll. Un. Mat. Ital. 8 (1973), 286-289.

18. __ Behnke-Stein theorem for analytic spaces, Trans. Amer. Math. Soc. 199 (1974), 317326.

19. G. Sorani, Homologie des q-paires de Runge, Ann. Scuola Norm. Sup. Pisa (3) 17 (1963), 319-332.

20. G. Sorani and V. Villani, q-complete spaces and cohomology, Trans. Amer. Math. Soc. 125 (1966), 432-448.

21. B. M. Weinstock, An approximation theorem for $\bar{\partial}$-closed forms of type $(n, n-1)$, Proc. Amer. Math. Soc. 26 (1970), 625-628.

Dipartimento di Matematica, Università di Roma "La Sapienza", I-00185 Roma, Italy 